\title{
A PSTN Terminal for FSK Decoding and DTMF Dialing Applications
}

\author{
Liang Xin-Tao, Fan Qing-Yu and ${ }^{1}$ Lin Wen-Chang \\ Affiliation: College of Electrical and Electronic Engineering, Harbin University of \\ Science and Technology, Harbin, 150080, China \\ ${ }^{1}$ E-mail:lxt3@163.com
}

\begin{abstract}
To realize Frequency Shift Keying (FSK) decoding and Dual-Tone Multi-Frequency (DTMF) dialing, a novel hardware and software solution is presented. These two signals are used to receive or send messages respectively in this solution which can meet different requirements of many Public Switched Telephone Network (PSTN) applications. The hardware used CPC5622 as the phone line interface Data access arrangement (DAA), HT9032D as the FSK decoder, and C8051f120 as the DTMF generator and system controller. The software, mainly composed of DTMF dialing and FSK decoding subprograms, was written and debugged with the hardware and finally succeeded to realize the terminal. The results performed under Huawei $C \& C 08$ switch show that the terminal can realize dialing by encoding standard DTMF signals and extracting the caller's information from the standard FSK signals in the PSTN. The CPC5622 as well as C8051f120 has some free Inputs, outputs, and other hardware resources, which make the PSTN terminal scalable and available to a variety of PSTN devices and applications.
\end{abstract}

Keywords: Public Switched Telephone Network (PSTN), Dual-Tone Multi-Frequency (DTMF), Frequency Shift Keying (FSK), Data access arrangement (DAA)

\section{Introduction}

With the advent of the Internet of Things era, people are able to control various home devices [1-3]. Meanwhile, the PSTN is around every corner of people's lives and work as the nervous system [4-5], which make the remote control system in smart home through the PSTN possible and economy.

CHEN (2012) showed a broadcast and remote control system in mine [6], in which DTMF signals are produced by the HT9200A, and the PSTN interface is realized by circuits instead of DAA. ZHAO (2014) demonstrated a kind of FSK Calling Information Collector [7], extracting the caller's information from the data decoded from the standard FSK signals in the PSTN by the HT9032D. LI (2014) showed an intelligent monitoring security control system based on PSTN network [8], coding and decoding DTMF signals by the MT8888. These applications have few free I/Os and are solutions without the DAA, so it is difficult to adapt them to other applications.

In accordance with the properties of Chinese PSTN, this paper presents an available to remote control, Cost-effective and scalable PSTN terminal, combined with C8051f120 microcontroller, CPC5622 DAA, HT9032D FSK decoder and other integrated circuits. We can send dialing digits with DTMF signals and receive Caller Identity (CID) data with FSK signals through the PSTN terminal. So the remote control systems in smart home and some PSTN applications can be accomplished easily via the PSTN terminal. 


\section{The Architecture of the PSTN Terminal}

As can be seen in Figure 1, the PSTN terminal can be divided into three main modules according to their functions, DAA module, FSK receiving module and C8051F120 microcontroller module.

The terminal is connected to the PSTN through DAA module via a telephone line. The /RING or /RING2 of the DAA module providing ringing detect pulses is connected to the /INT1 of the C8051f120 as the source of the ring counter. The DAA module also provides $\mathrm{AC}$ and DC phone line terminations, on/off-hook switch, full time receive on-hook transmission capability and 2-wire to 4-wire hybrid including a 2-wire TX and a 2-wire $\mathrm{RX}$. The TX is connect to the C8051f120's A/D, and the RX is connect to the C8051f120's D/A, where DTMF dialing is completed by the program.

From the telephone lines directly or from the DAA module, FSK receiving module achieve the FSK signals, which are demodulated to binary data to be sent to the MCU module.

The MCU module should monitor the rings from the /RING or /RING2 to make controls. The FSK receiving program is started by the MCU module after the first ringing, and ended when the receiving completes or the receiving time expires. Except for receiving FSK CID, another main function of the PSTN terminal is to send DTMF dialing. MCU module must put the DAA module in the on-hook condition by the $/ \mathrm{OH}$ control input before sending DTMF signals. Under the control of the DTMF program, the internal DAC of the MCU module generates the DTMF signals, which are coupled to the telephone line to make a call by the DAA module.

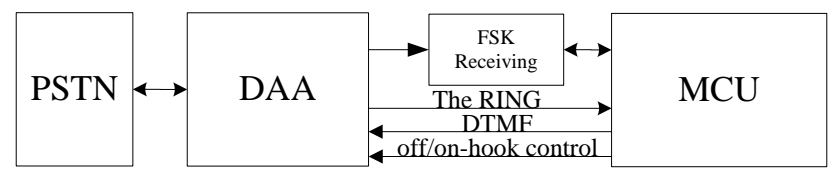

Figure 1. Diagram of the System

\section{Hardware Design of the PSTN Terminal}

\subsection{MCU Module}

The mixed-signal ISP FLASH microcontroller C8051F120 of the Silicon labs is selected as the host controller of the hardware system. The main functions of the C8051F120 in the PSTN terminal include monitoring the rings from the /RING or /RING2, placing the DAA in on-hook or off-hook state, generating DTMF signals by the internal DAC, extracting the caller's information from the data received from the HT9032D, computing, and controlling the system etc.

\subsection{DAA Module}

The CPC5622 LITELINK III of IXYS Corporation is selected as Silicon DAA. The CPC5622 provides half-wave and full-wave ringing detection by/RING or /RING2, on/off-hook control by /OH, and 2-wire phone line to 4-wire hybrid, a 2-wire TX and a 2wire RX in the PSTN terminal. The CPC5622 also provides AC and DC phone line terminations and full time receive on-hook transmission capability [9], which makes the terminal can be further extended.

\subsection{FSK Receiving Module}

FSK Receiving Module is made up by the HT9032D designed for receiving physical layer signals transmitted according to Bell core TR-NWT-000030 and ITU-TV.23 specifications. The telephone line is connected to TIP and RING pins of the HT9032D by 
a resistor network to attenuate the incoming power ring. FSK signals are transmitted between the first time and the second time power ring. The HT9032D will demodulate the FSK signals $($ Logical 1 (Mark) $=1200+/-12 \mathrm{~Hz}$, Logical $0($ Space $)=2200+/-22 \mathrm{~Hz}$ for mode 1 of the Bell 202 or Logical 1 (Mark) $=1300 \mathrm{~Hz}$, Logical 0 (Space) $=2100 \mathrm{~Hz}$ for mode 2 of the V.23, the transmission rate of 1200bps in both modes) to serial asynchronous binary data after the first valid ring. These data include channel occupancy signals, flag signals, as well as all the caller identification signals, which are exported from the DOUT pin.

\section{Programming}

DTMF signals are generated by the 12 bits DAC of the C8051F120. The amplitudes of the DTMF signals are searched in a sine Table according to the phase values, calculated by the C8051F120 depending on the frequency of the DTMF signals. The phase calculation formula is as follow:

PHASE $=t * f *$ PHASE_PRECISION/SAMPLE_RATE_DAC

The value $t$ is the number of times counting the overflows of the timer for the frequency of the DAC output. The value $\mathrm{f}$ is the frequency of the digit according to the ITU-T Q.23 [10]. The sampling rate of DAC is set to $100000 \mathrm{~Hz}$ (SAMPLE_RATE_DAC $=100000)$ and the DAC phase precision for calculation is set to 65536 (DACPHASE_PRECISION= 65536).

Because we use a sine Table of 256 points and 16 bits precision, we need to divide the accumulated phase by 256 before Look-up the sine Table.

According to the ITU-T Q.24 [11], there are ten DTMF dials per second at most (Processing time interval $<100 \mathrm{~ms}$ ), a DTMF signal duration is more than $45 \mathrm{~ms}$ and less than $55 \mathrm{~ms}$ (Required signal time duration $45 \mathrm{~ms}<\mathrm{TS}<55 \mathrm{~ms}$ ), and minimum pause durations between DTMF signals are more than $45 \mathrm{~ms}$ (Silence time interval $>45 \mathrm{~ms}$ ). In this Terminal, we use a program of variable delay to control state durations, the signal duration is set to $130 \mathrm{~ms}$ and the silence time interval is set to $140 \mathrm{~ms}$.

Programming was completed in the Silicon Labs Integrated Development Environment (IDE) development environment, mainly based on the CIP-51 microcontroller fully compatible with the MCS-51 $51^{\mathrm{TM}}$ instruction set. The program mainly consists of an initialization subprogram which configures Crossbar, System Clock, interrupts, timers, DAC, ADC, a DTMF dialing subprogram and a FSK decoding subprogram etc. The following describes the realization of the latter two subprograms.

The DTMF dialing subprogram is to dial the telephone number digit by digit. For each digit in the number, two phases are first added up by DAC sampling rate respectively based on the two frequencies of the digit corresponding to the ITU-T Q.23 [10]. The amplitude is added up by the two amplitudes, found in the sine Table according to the two phases, and sent to the DAC continuously to make the DTMF tone. Each DTMF tone for a digit lasts for $130 \mathrm{~ms}$, stops for $140 \mathrm{~ms}$ and then start to send next digit until the last digit is sent. DTMF dialing program works according to the following flow chart of Figure 2. 


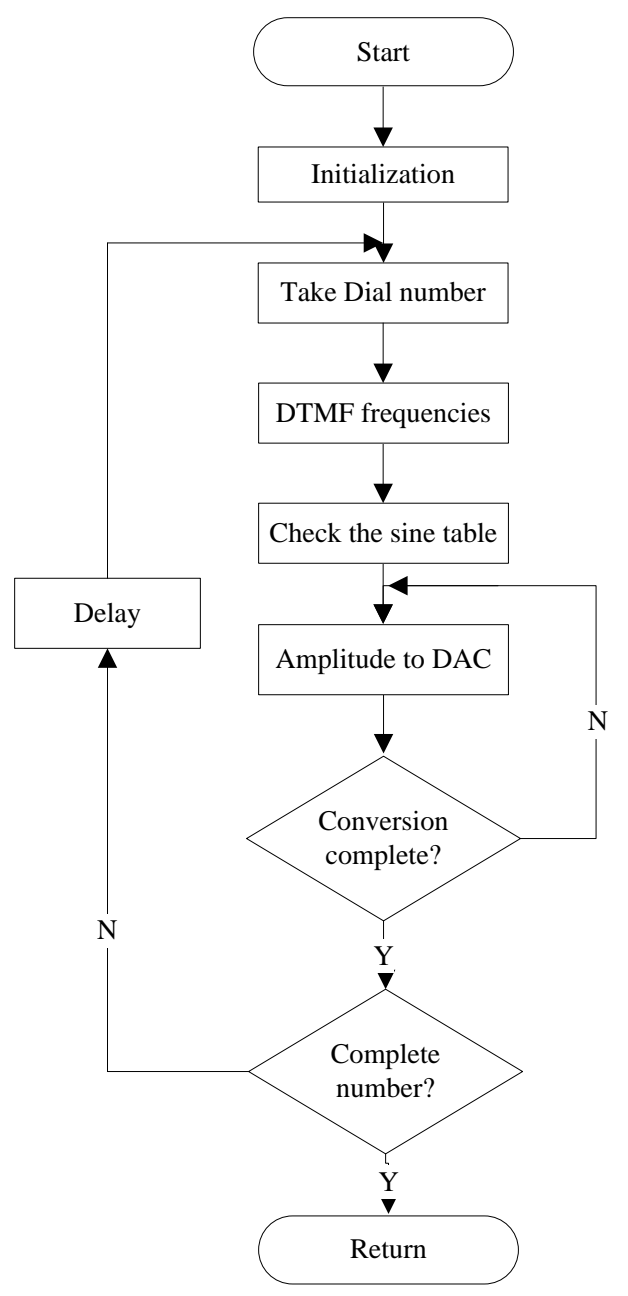

Figure 2. Flow Chart of the DTMF Subprogram

The FSK decoding subprogram is to get the caller's number, date and other caller information from the serial data sent by the HT9032D. There are two kinds of Caller identification information data formats: Single Data Message Format (SDMF) and the Multiple Data Message Format (MDMF). The headers of the two data formats are all channel occupancy signals composed of a group of 300 consecutive alternate 0 and 1 (binary bits). Message type byte, following the channel occupancy signals, is " $04 \mathrm{H}$ "in the SDMF and "80H" in the MDMF. Total length of the message byte follows the message type byte. Time and the calling number messages follow directly in the SDMF, and there are delimiter $01 \mathrm{H}$ and the length of time message before Time message, delimiter $02 \mathrm{H}$ and the length of the calling number message before the calling number message in the MDMF. Thus FSK subprogram detects and counts 0X55 (01010101) as channel occupancy signals in the serial data, then gets message type byte, $04 \mathrm{H}$ or $80 \mathrm{H}$, to extract the time and calling number messages in accordance with the data format. FSK decoding subprogram works according to the following flow chart of Figure 3. 


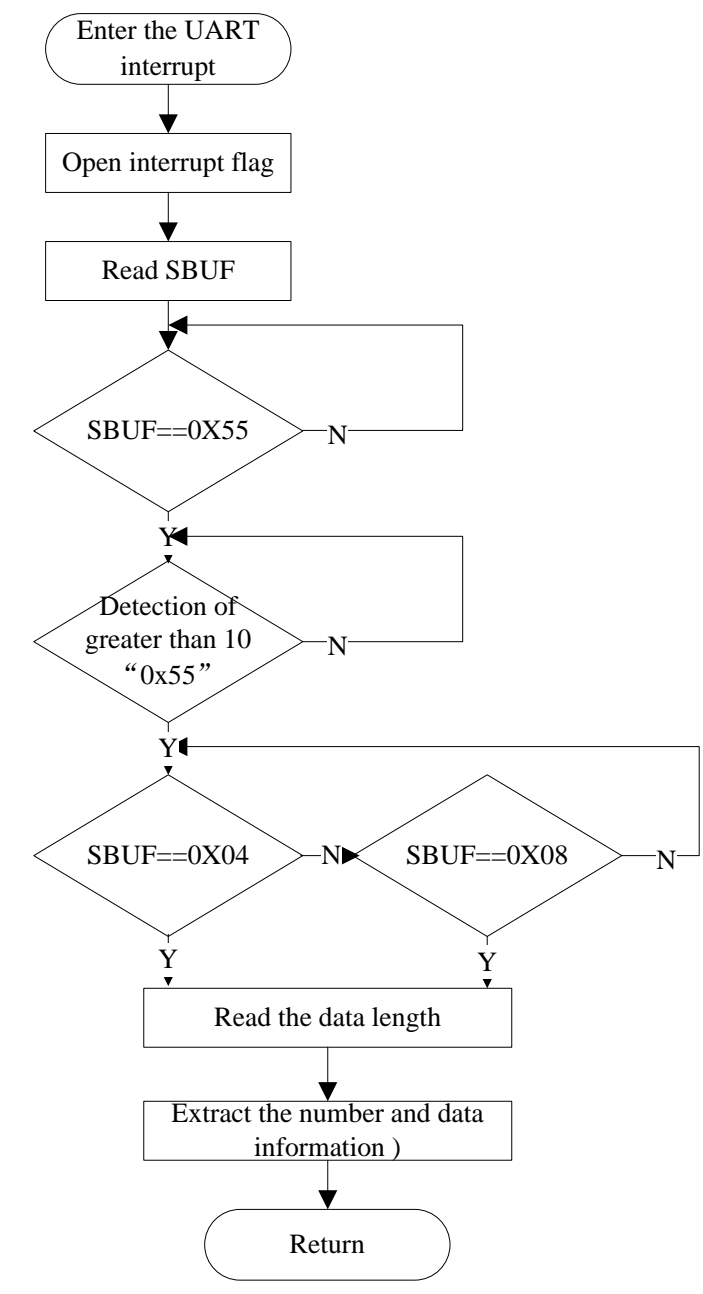

Figure 3. Flow Chart of the FSK Subprogram

\section{Experimental Results and Analysis}

In order to debug and verify the functions of the terminal, we carry out the experiments on the two subprograms under the experimental environments of the C\&C08 switch and the HJD-80 switch, respectively. The results show that the PSTN terminal can successfully dial the number of the telephone and get the caller's information using the two subprograms under both environments. We use storage oscilloscope to record the result waveforms, and Silicon Labs IDE online simulation to verify decoding results of the FSK CID. Some of the results under the C\&C08 switch are as follows.

Figure 4 shows the DTMF waveforms caught by the digital storage oscilloscope. The telephone of "5550026" rings when the PSTN terminal dial the number "5550026", which proves that the PSTN terminal can dial the number of the telephone via DTMF signals. The PSTN terminal can also send other data via DTMF signals in the communication. 


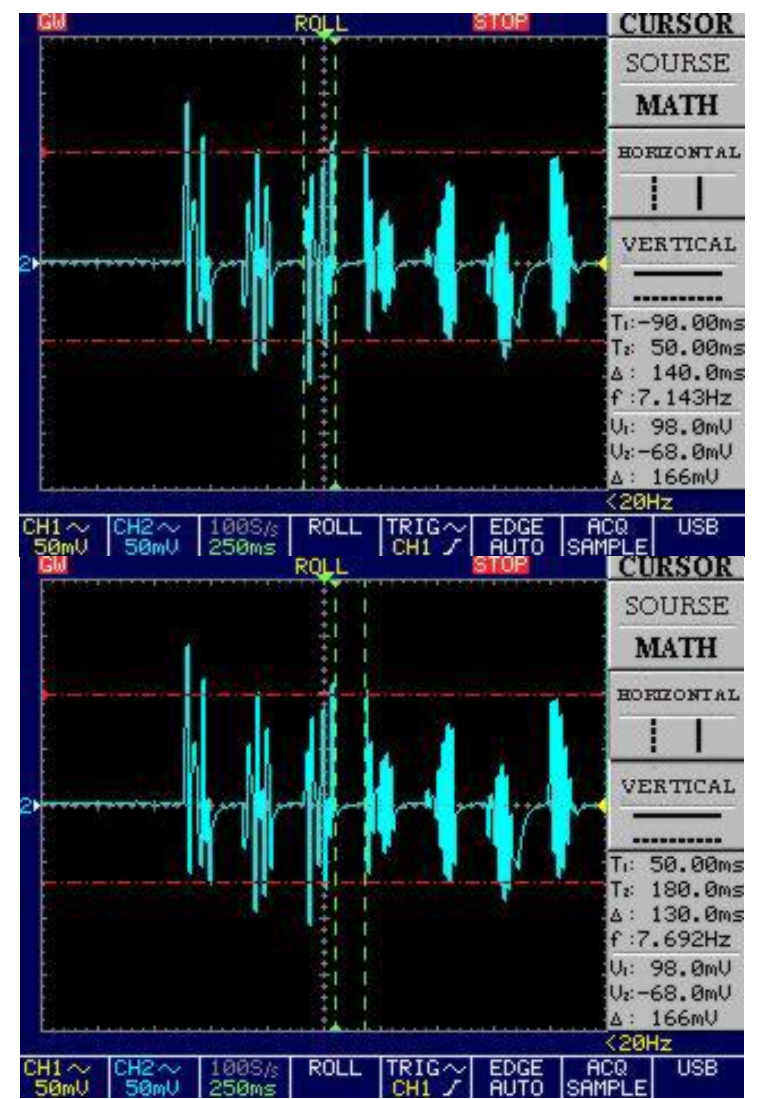

\section{Figure 4. The Dialing Waveforms of DTMF Signals}

Figure 5 shows the FSK signal waveforms on the telephone line when there is a call into the PSTN terminal. The FSK waveforms appear after the first ringing signal, because of the high volt of the power ring signal $(90 \pm 15 \mathrm{~V})$ the oscilloscope can only capture the temporal envelope of the FSK signals.

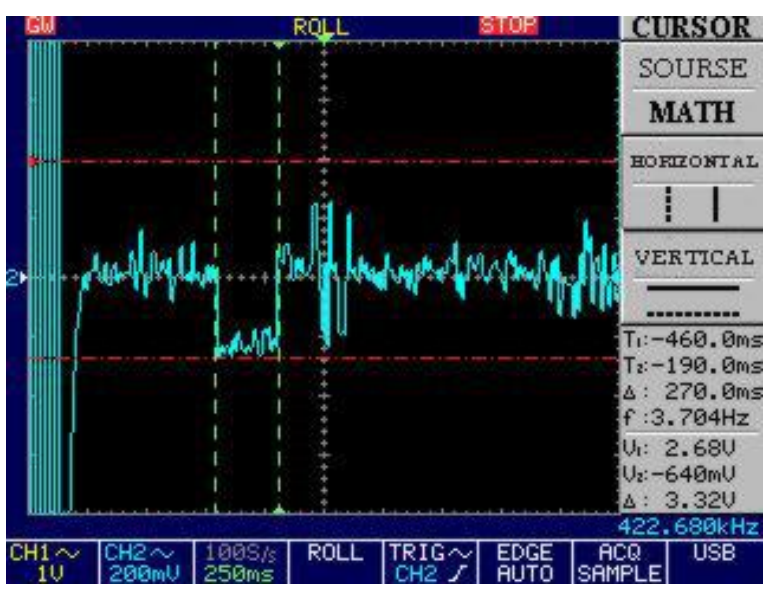

Figure 5. The Waveforms of FSK Signals

Figure 6 shows the results of the FSK decoding. We can clearly see channel occupancy signals $(0 \times 55=01010101)$, the message type byte $(0 \times 80)$ in the MDMF, the total length of the message byte $(0 \times 13)$, the delimiter $(0 x 01)$, the time message length $(0 x 08)$, the time message (at 15:52 on October 27), the delimiter (0x02), the calling number message length (0x07), the calling number message (5550020). 
Figure 7 shows the $\mathrm{C} \& \mathrm{C} 08$ experimental environment including the distribution frame, the BAM server, and the C\&C08 switch. The C\&C08 switch is configured to provide services to meet the needs of the experiments via the BAM server. The C\&C08 can provide nearly all services of a municipal telephone station in the laboratory environment.

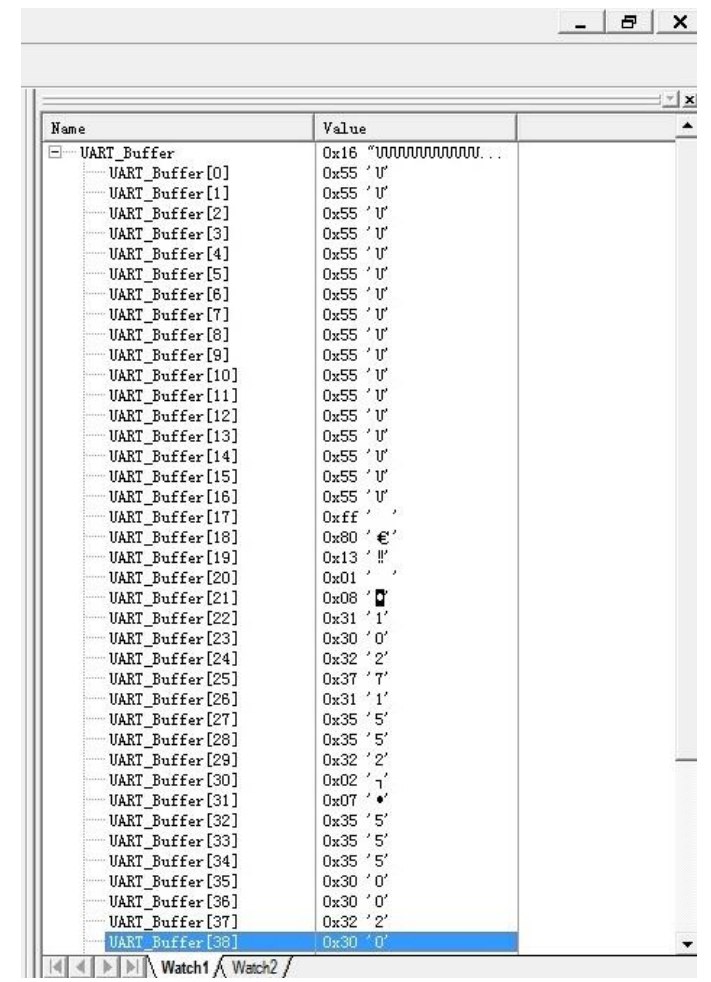

Figure 6. The Results of FSK Decoding

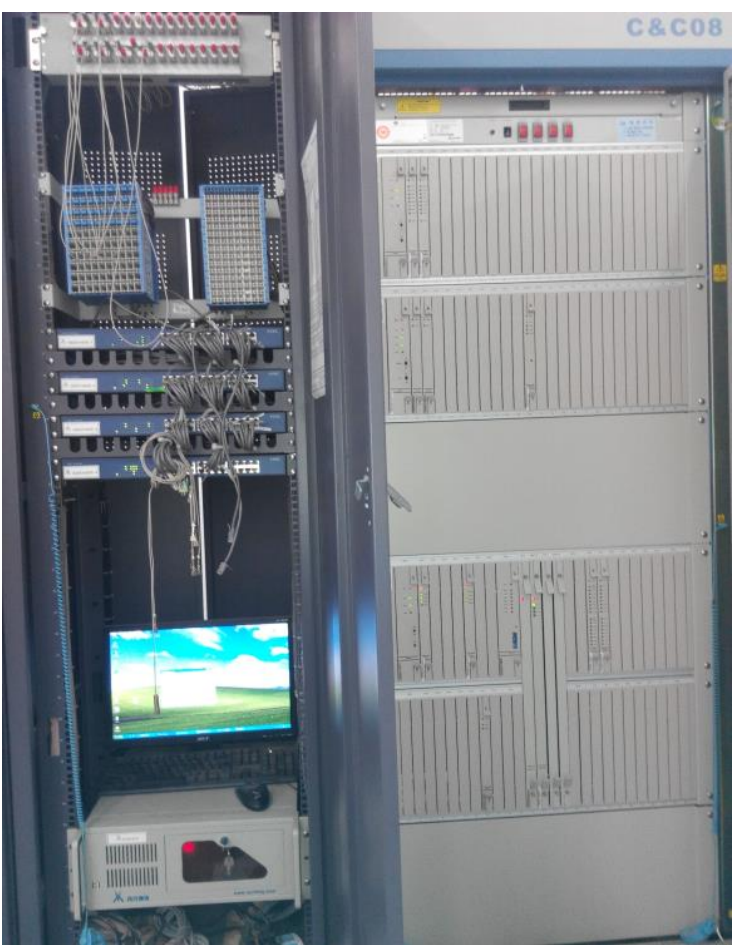

Figure 7. The C\&c08 Switch 


\title{
6. Conclusion
}

The terminal can realize ringing detection, on/off-hook control, FSK calling information extraction, DTMF dialing and so on. Because of 2 -wire phone line to 4wire hybrid feature of the CPC5622, the terminal can be extended and used in other voice and data communication applications. C8051F120 is a high speed 8-bit microcontroller with many free hardware resources and can be used in many applications, such as network. In short, the terminal is a simple solution to realize the FSK signals decoding and DTMF signals dialing in the PSTN and has the capabilities to be adapt to other PSTN applications.

\section{Acknowledgement}

Project supported by the Natural Science Foundation of Heilongjiang Province (Grant No.QC2014C075), Harbin innovation talents Research Fund (Grant No. RC2013LX007003) and the Innovation and Entrepreneurship Training Project for College Students of Heilongjiang Province (Grant No. 201310214052).

\section{REFERENCE}

[1] K. Xiang-Zhe, "Talking about the current situation and development trend of China's smart home", China Science and Technology Information, no. 8,(2014), pp. 121-122.

[2] M. Xi-Ren and X. Li-Long, "The current situation and development of China's Intelligent home", Intelligent Building, (2010).

[3] Z. Yong-Gang, "The current situation and problems of China's Intelligent home", Intelligent Buildings and Urban Information, no. 12, (2012).

[4] Z. Gang, "Design of household appliances control system based on PSTN", Journal of Mongolia Normal University: Natural Science Edition, vol. 42, no. 4, (2013), pp. 428-430.

[5] W. Wei, F. Nan and X. Ke-Qing, "Design of a water-level monitoring system of the remote reservoir based on PSTN", Electronic Design Engineering, vol. 18, no. 12, (2010), pp. 178-180.

[6] C. Dong-Dong, T. Shi-Jun and G. Min-Ming, "Broadcast used in mine and remote control system based on FSK and DTMF", Journal of Mechanical \& Electrical Engineering, vol. 29, no. 1, (2012), pp. 108111.

[7] Z. Yi-Ran, C. Wei and Z. Guang-Yuan, "The Research and Design of FSK Calling Information Collector", Computer \& Telecommunication, vol. 3, (2014), pp. 26-28.

[8] L. Mu-Yun, W. Wei-Xing, L. Hao-Ming and Z. Da-Chi, "Design of intelligent monitoring security control system based on PSTN network control", Electronic Design Engineering, no. 6, (2014), pp. 7-9.

[9] "IXYS Integrated Circuits Division", CPC5622 LITELINK® III Phone Line Interface IC (DAA) $[\mathrm{DB} / \mathrm{OL}]$.

[10] http://www.ixysic.com/home/pdfs.nsf/www/CPC5622.pdf/\$file/CPC5622.pdf.

[11] International Telecommunication Union, "Technical features of pushbutton telephone sets," Recommendation Q.23, Telecommunication Standardization Sector of ITU, Geneva, Switzerland, (1988).

[12] International Telecommunication Union, "Multifrequency push-button signal reception," Recommendation Q.24, Telecommunication Standardization Sector of ITU, Geneva, Switzerland, (1988).

\begin{abstract}
Authors
Liang Xin-Tao, He received his M.Sc. in signal and information processing (2006) from Harbin Institute of Technology. Now he is associate professor of College of Electrical and Electronic Engineering, Harbin University of Science and Technology. His current research interests include signal processing technology and VOIP technology.
\end{abstract}

